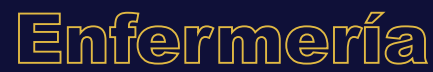

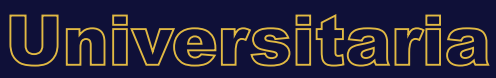

\section{Características sociodemográficas y clínicas de pacientes con disfunción o con rechazo de injerto renal}

\section{Clinical and sociodemographic characteristics of patients with kidney dysfunction or kidney transplant rejection}

\section{Características sociodemográficas e clínicas de pacientes com disfunção ou rejeição do enxerto renal}

\author{
R.M. Rodríguez-Medina ${ }^{a 1,2^{*}}$, C.F. Meza- \\ García $^{\mathrm{b} 1,2}$, N.Y. Díaz-García ${ }^{\mathrm{c} 1,2}$ \\ ORCID \\ a $\underline{0000-0003-4216-7987}$ \\ b $\underline{0000-0002-9853-227 X}$ \\ d $\underline{0000-0003-2045-9962}$ \\ ${ }^{1}$ Hospital Regional de Alta Especialidad del Bajío, Subdirección de Enfermería, Guanajuato, \\ México \\ ${ }^{2}$ Universidad de Guanajuato, Departamento de Enfermería y Obstetricia, Guanajuato, México
}

Recibido: 13 septiembre 2019

Aceptado: 26 junio 2020

RESUMEN

Introducción: La identificación de las características sociodemográficas y clínicas de los pacientes con disfunción o con rechazo del injerto renal proporcionará información para dirigir la aplicación de intervenciones de enfermería que contribuyan a aumentar la supervivencia del paciente y del injerto renal.

\footnotetext{
*Autora para correspondencia. Correo electrónico: anidem_2_2@hotmail.com 
Objetivo: Describir las características sociodemográficas y clínicas de los pacientes con disfunción o con rechazo del injerto renal de un hospital de tercer nivel de León, Guanajuato. Métodos: Estudio retrospectivo y descriptivo, muestreo por conveniencia. Se identificaron 118 expedientes clínicos de pacientes adultos con diagnóstico de disfunción o con rechazo de injerto renal durante el periodo 2016-2018. Se compararon datos sociodemográficos y clínicos de ambos grupos de pacientes. Se consideró un valor de $p<0.05$ para la significancia estadística.

Resultados: El 20.3\% de los pacientes ingresaron por disfunción y 79.7\% por rechazo de injerto. La edad media de los pacientes fue de $29.9 \pm 10.2$ años con un rango de edad considerado entre 20 a 84 años. La mayoría de los pacientes fueron hombres con antecedente de trasplante renal de donante fallecido. No se encontraron diferencias en las características clínicas y sociodemográficas de los pacientes con disfunción y los de rechazo de injerto renal.

Conclusiones: La disfunción y el rechazo de injerto renal continúan siendo problemas frecuentes. Aunque no es el caso del presente estudio, se ha encontrado asociación entre algunas características sociodemográficas y la presencia de complicaciones posteriores al trasplante renal.

Palabras clave: Trasplante; rechazo de injerto; enfermería; México.

\section{ABSTRACT}

Introduction: The identification of clinical and sociodemographic characteristics of patients with kidney dysfunction or kidney transplant rejection can help the design and implementation of nursing interventions which contribute to the patient's survival.

Objective: To describe the clinical and sociodemographic characteristics of patients suffering from kidney dysfunction or kidney transplant rejection in a 3rd level hospital in the city of Leon, Guanajuato, Mexico.

Methods: This is a retrospective and descriptive study using sampling by convenience. 118 clinical records of adult patients with a diagnosis of kidney dysfunction or kidney transplant rejection during the period 2016-2018 were identified. Clinical and sociodemographic data from both groups of patients were compared. A value of $p<0.05$ was accepted as statistically significant.

Results: $20.3 \%$ of the total patients were diagnosed with kidney dysfunction and $79.7 \%$ with kidney transplant rejection. The mean patient age was $29.9 \pm 10.2$ years old with a range of 20 to 84 . The majority of patients were male with a background of having received a kidney transplant from a deceased donor. No main differences in clinical and sociodemographic characteristics were found between the patients with kidney dysfunction and the patients with kidney transplant rejection.

Conclusions: Kidney dysfunction and kidney transplant rejection continue being frequent health problems, and a better knowledge of the clinical and sociodemographic characteristics of these patients, even if no main differences between the two groups are found, will contribute to the better design and implementation of the related nursing interventions.

Keywords: Transplantation; graft rejection; nursing; Mexico. 


\section{RESUMO}

Introdução: A identificação das características sociodemográficas e clínicas dos pacientes com disfunção ou com rejeição do enxerto renal proporcionará informação para dirigir a aplicação de intervenções de enfermagem que contribuam para aumentar a sobrevivência do paciente e do enxerto renal.

Objetivo: Descrever as características sociodemográficas e clínicas dos pacientes com disfunção ou com rejeição do enxerto renal de um hospital de terceiro nível de León, Guanajuato.

Métodos: Estudo retrospectivo e descritivo, amostragem por conveniência. Identificaram-se 118 expedientes clínicos de pacientes adultos com diagnóstico de disfunção ou com rejeição do enxerto renal durante o período 2016-2018. Compararam dados sociodemográficos e clínicos de ambos os grupos de pacientes. Considerou-se um valor de $p<0.05$ para a significância estatística.

Resultados: O 20.3\% dos pacientes ingressaram por disfunção e 79.7\% por rejeição do enxerto. A média de idade dos pacientes foi de $29.9 \pm 10.2$ anos com uma faixa etária de 20 a 84 anos. A maioria dos pacientes foram homens com antecedente de transplante renal de doador falecido. Não se encontraram diferenças nas características clínicas e sociodemográficas dos pacientes com disfunção e os de rejeição do enxerto renal.

Conclusões: A disfunção e a rejeição do enxerto renal continuam sendo problemas frequentes. Embora não seja o caso do presente estudo, foi encontrada associação entre algumas características sociodemográficas e a presença de complicações após o transplante renal.

Palavras chave: Transplante; rejeição de enxerto; enfermagem; México.

\section{INTRODUCCIÓN}

En el continente americano la enfermedad renal crónica (ERC), como consecuencia de la diabetes y la hipertensión, representa un problema de salud pública de carácter prioritario, ya que se estima una prevalencia de 1,142 personas con ERC y una incidencia de 377 casos por millón de habitantes. Por otra parte, los años de vida ajustados por discapacidad de los pacientes con ERC aumentaron un 58\% en América Latina desde 1990 hasta 2010².

Actualmente, el trasplante renal (TR) es el tratamiento de elección para los pacientes con ERC terminal. En el 2019, de acuerdo con el Centro Nacional de Trasplantes (CENATRA), los tres estados con mayor número de trasplantes renales fueron la Ciudad de México con 839, Jalisco con 598 y el estado de Guanajuato con 207 trasplantes³. No obstante, se ha evidenciado una mayor calidad de vida del paciente que ha recibido un trasplante y mejores resultados a corto plazo debido a los avances en la terapia inmunosupresora y las técnicas de diagnóstico; por el contrario, los resultados a largo plazo continúan amenazando la supervivencia del injerto y del paciente mismo ${ }^{4-6}$.

Al respecto, se ha identificado a la disfunción crónica del injerto renal como la causa más frecuente de muerte del paciente con injerto funcional y de pérdida de injerto con una tasa anual aproximada del 3 al 5\%7. Cabe destacar que la causa principal de disfunción es el rechazo de injerto renal, el cual presenta una incidencia del 5 al 10\% y puede aumentar hasta 35\% en pacientes de alto riesgo inmunológico ${ }^{8}$. 
Estudios previos han identificado factores relacionados con la presencia de disfunción y rechazo de injerto renal. Campistol et al., observaron que la disfunción crónica del injerto renal está asociada con diversos factores como el donador fallecido, el tiempo prolongado de isquemia fría (periodo de tiempo que transcurre entre el cese de la circulación sanguínea del donante y su implante vascular en el receptor) y características del receptor como la presencia de anemia7. Por su parte Etta et al., identificaron factores asociados con el rechazo de injerto renal, los cuales incluyen la presencia de anticuerpos específicos del donador, donante mayor de 60 años, tiempo prolongado de isquemia fría, retraso en la función del injerto y la falta de adherencia al tratamiento inmunosupresor 9 .

En este contexto es necesario que se consideren los factores antes mencionados para desarrollar estrategias dirigidas a disminuir la tasa de pérdida de injerto renal ${ }^{10}$. El personal de enfermería desempeña un papel clave interviniendo de manera activa en la educación sanitaria del paciente que ha recibido un trasplante, con el objetivo de mejorar los resultados de este. Como ejemplos encontramos el estudio de Ruíz et al., y Durán et al., para aumentar la adherencia al tratamiento inmunosupresor posterior al $\mathrm{TR}^{11,12}$.

Dados los argumentos expuestos, se planteó el presente estudio con el objetivo de describir las características sociodemográficas y clínicas de los pacientes que se diagnosticaron con disfunción o con rechazo de injerto renal en un hospital de tercer nivel de la ciudad de León, Guanajuato durante el periodo 2016-2018. La hipótesis que se formuló fue que los pacientes con disfunción y los de rechazo de injerto renal mostraban diferencias en sus características sociodemográficas y clínicas. Lo anterior proporcionará información para dirigir la aplicación de estrategias e intervenciones de enfermería que contribuyan a aumentar la supervivencia del paciente y del injerto renal.

\section{MÉTODOS}

El presente es un estudio de tipo retrospectivo y descriptivo. La unidad de análisis fueron los expedientes clínicos de pacientes con diagnóstico de disfunción o con rechazo de injerto renal, los cuales ingresaron al servicio de trasplantes de una institución de tercer nivel de atención en la ciudad de León, Guanajuato durante el periodo 2016-2018. Se calculó el tamaño de la muestra para proporciones con el software EPIDAT 3.1. Además, se realizó un muestreo no probabilístico y por conveniencia, se incluyeron 118 expedientes que cumplieron con los criterios de inclusión, es decir, registros clínicos de pacientes de ambos sexos, mayores de 18 años y con antecedente de TR. Los criterios de exclusión fueron expedientes con registros clínicos incompletos de las variables sociodemográficas y clínicas que se incluyeron en nuestro estudio.

La recolección de datos se realizó durante el periodo de enero a marzo de 2019, por personal de enfermería capacitado que previamente firmó una carta de confidencialidad. El diagnóstico de disfunción o de rechazo de injerto fue el reportado en el expediente clínico. La disfunción crónica del injerto se definió como aquella complicación que se presenta después de 6 meses de realizado el TR con un deterioro lento y progresivo de la función renal (tasa de filtración glomerular $<40 \mathrm{ml} / \mathrm{min} / 1.73$ $\left.\mathrm{m}^{2}\right)^{13} \mathrm{y}$ manifestado clínicamente por hipertensión y proteinuria $>300 \mathrm{mg}$ por día ${ }^{14}$. El rechazo de injerto renal incluyó al rechazo celular y humoral, cuyo diagnóstico es histológico de acuerdo con los criterios de Banff. Clínicamente los pacientes con rechazo de injerto cursan con un aumento en la creatinina sérica, hipertensión y proteinuria ${ }^{15}$.

Las características sociodemográficas describen la edad, sexo, estado civil, escolaridad, ocupación y nivel socioeconómico del paciente. Las características clínicas se operacionalizaron para describir la historia clínica del paciente, la cual incluye antecedentes heredofamiliares, etiología de la ERC, 
terapia de reemplazo renal previa al trasplante, toxicomanías, presencia o ausencia de hacinamiento, composición corporal (peso, talla, IMC) y por último, antecedentes relacionados con el TR, tipo de donador vivo o fallecido, parentesco con el donador (en caso de donante vivo relacionado), tiempo transcurrido desde el trasplante y el tiempo de isquemia fría.

Para el análisis estadístico se utilizó el programa SPSS versión 21. Las variables cualitativas se reportaron con frecuencias y porcentajes, mientras que las variables cuantitativas se presentaron con media y desviación estándar. Se evaluó la normalidad en la distribución de los datos mediante la prueba de bondad de ajuste de Kolmogorov-Smirnov con corrección de Lilliefors. Se compararon las características clínicas y sociodemográficas de los pacientes con disfunción y los de rechazo de injerto renal a través de la prueba t de Student para muestras independientes y la prueba chi cuadrada. Se aceptó un valor de $p<0.05$ para la significancia estadística.

El protocolo de investigación fue aprobado por el Comité de investigación y el Comité de ética en investigación de la institución de salud. Se cumplió con los lineamientos de la Declaración de Helsinki año $2013^{16}$ y el Reglamento de la Ley General de Salud en materia de investigación para la salud en su versión vigente. De acuerdo con el artículo 17 se consideró un estudio con riesgo mínimo ${ }^{17}$.

\section{RESULTADOS}

Se identificaron 118 expedientes clínicos de pacientes adultos que ingresaron al servicio de trasplantes durante el periodo 2016-2018 con diagnóstico de disfunción (20.3\%) y rechazo de injerto renal (79.7\%). La edad media de los pacientes fue de $29.9 \pm 10.2$ años con un rango de edad de 20 a 84 años. En la tabla 1 se presentan las características sociodemográficas de los pacientes con disfunción y los de rechazo de injerto renal. En ambos grupos la mayoría de los pacientes fueron hombres, solteros, con secundaria terminada y nivel socioeconómico medio-bajo de acuerdo con el reporte de gerencia social.

Asimismo, en la tabla 2 se presenta información sobre la historia clínica de los pacientes de ambos grupos. Cabe mencionar que, aunque no se encontraron diferencias entre las características estudiadas y la presencia de disfunción o rechazo de injerto, la mayoría de los pacientes de ambos grupos mostraron características similares como son: etiología de la enfermedad renal no determinada durante el interrogatorio médico, diálisis y hemodiálisis previas al TR, toxicomanías negadas y vivir en condiciones de hacinamiento.

Por otra parte, no se encontró asociación entre las características del donador (tipo de donador y parentesco con el receptor) y la presencia de disfunción o de rechazo de injerto renal. Además, el tiempo transcurrido desde el trasplante y el tiempo de isquemia fría no mostraron diferencias en ambos grupos (Tabla 3).

Finalmente, a partir de los resultados de estudios previos se formuló la hipótesis de la posible relación entre las características sociodemográficas y clínicas con la presencia de disfunción o de rechazo de injerto renal. Para ello se realizó un análisis de regresión en donde no se encontró asociación entre los factores mencionados (introducidos como factores predictores) y la presencia de complicaciones (resultados no mostrados), por lo que no se cuenta con evidencia suficiente para aceptar la hipótesis planteada.

\section{DISCUSIÓN}

En el presente estudio no se observaron diferencias en las características sociodemográficas y clínicas de los pacientes diagnosticados con disfunción y rechazo de injerto renal en un hospital de tercer 


\begin{tabular}{|c|c|c|c|c|c|}
\hline \multirow{2}{*}{$\begin{array}{l}\text { Edad (años)* } \\
\text { Media (DE) }\end{array}$} & \multicolumn{2}{|c|}{$\begin{array}{c}\text { Disfunción }(n=24) \\
f(\%)\end{array}$} & \multicolumn{2}{|c|}{$\begin{array}{c}\text { Rechazo }(n=94) \\
f(\%)\end{array}$} & \multirow{2}{*}{$\begin{array}{l}\text { Valor } p \\
0.473\end{array}$} \\
\hline & $28.5 \pm$ & 5.8 & $30.2 \pm$ & 11.1 & \\
\hline $\begin{array}{l}\text { Sexo } \\
\text { Masculino } \\
\text { Femenino }\end{array}$ & $\begin{array}{r}17 \\
7\end{array}$ & $\begin{array}{l}(70.8) \\
(29.2)\end{array}$ & $\begin{array}{l}72 \\
22\end{array}$ & $\begin{array}{l}(76.6) \\
(23.4)\end{array}$ & 0.558 \\
\hline $\begin{array}{l}\text { Estado civil } \\
\text { Soltero } \\
\text { Casado }\end{array}$ & $\begin{array}{r}17 \\
7\end{array}$ & $\begin{array}{l}(70.8) \\
(29.2)\end{array}$ & $\begin{array}{l}65 \\
29\end{array}$ & $\begin{array}{l}(69.1) \\
(30.9)\end{array}$ & 0.873 \\
\hline $\begin{array}{l}\text { Escolaridad } \\
\text { Ninguno } \\
\text { Primaria } \\
\text { Secundaria } \\
\text { Bachillerato } \\
\text { Licenciatura }\end{array}$ & $\begin{array}{r}0 \\
0 \\
14 \\
8 \\
2\end{array}$ & $\begin{array}{r}(0.0) \\
(0.0) \\
(58.3) \\
(33.3) \\
(8.3)\end{array}$ & $\begin{array}{r}2 \\
15 \\
36 \\
30 \\
11\end{array}$ & $\begin{array}{r}(2.1) \\
(16.0) \\
(38.3) \\
(31.9) \\
(11.7)\end{array}$ & 0.174 \\
\hline $\begin{array}{l}\text { Ocupación } \\
\text { Hogar } \\
\text { Trabaja } \\
\text { Estudia } \\
\text { Desempleado }\end{array}$ & $\begin{array}{r}4 \\
11 \\
5 \\
24\end{array}$ & $\begin{array}{l}(16.7) \\
(45.8) \\
(16.7) \\
(20.8)\end{array}$ & $\begin{array}{r}8 \\
46 \\
19 \\
21\end{array}$ & $\begin{array}{r}(8.5) \\
(48.9) \\
(20.2) \\
(22.3)\end{array}$ & 0.698 \\
\hline $\begin{array}{l}\text { Nivel socioeconómico } \\
\text { Bajo } \\
\text { Medio } \\
\text { Alto }\end{array}$ & $\begin{array}{r}12 \\
12 \\
0\end{array}$ & $\begin{array}{r}(50.0) \\
(50.0) \\
(0.0)\end{array}$ & $\begin{array}{r}78 \\
16 \\
0 \\
\end{array}$ & $\begin{array}{r}(83.0) \\
(17.0) \\
(0.0)\end{array}$ & 0.061 \\
\hline
\end{tabular}

Comparación de frecuencias a través de la prueba chi cuadrada.

*Se presenta como media y desviación estándar.

nivel durante el periodo 2016-2018, por lo tanto, no se identificaron factores predictores de ambas complicaciones. Sin embargo, en otros estudios previos han encontrado asociación entre el tiempo de isquemia prolongado y la función retardada del injerto (FRI) ${ }^{18 .}$ Al respecto Hansson et al. ${ }^{19}$, afirman que el riesgo de pérdida de injerto después de 5 años del TR aumenta si el tiempo de isquemia fría excede las 14 horas, debido a la reducción de la masa renal funcional, la lesión vascular y la hipoxia crónica ${ }^{20}$.

Por otra parte, se ha observado que los pacientes fumadores ${ }^{21} \mathrm{y}$ con obesidad ${ }^{22}$ presentan menores tasas de supervivencia después del TR. En el presente estudio la mayoría de los participantes negó la presencia de toxicomanías y presentaron un IMC $<25 \mathrm{Kg} / \mathrm{m}^{2}$, las cuales son condiciones favorables. Al respecto Liese et al., encontraron que el IMC $>30 \mathrm{Kg} / \mathrm{m}^{2}$ se asoció con mayor frecuencia de infección de la herida quirúrgica, la función retardada de injerto y menor tasa de filtración glomerular a los 12 meses después del TR. Los hallazgos anteriores representan un área de oportunidad para el personal de enfermería y la necesidad de fomentar la reducción de peso para mejorar los resultados del TR²3.

También es importante conocer la ocupación de los pacientes después del TR. Tzvetanov et al. ${ }^{24}$, encontraron que la situación laboral en el periodo posterior al trasplante se asoció con la función del injerto y la supervivencia del paciente. En este sentido, se sugiere ofrecer apoyo psicológico temprano con el fin de asistir a los pacientes durante el proceso de adaptación y fragilidad psicológica posterior al trasplante para que puedan adaptarse de la mejor manera a las nuevas condiciones que ofrece el TR ${ }^{25}$. 
Tabla 2. Historia clínica de los pacientes con disfunción o rechazo de injerto renal

\begin{tabular}{|c|c|c|c|c|c|}
\hline Variable & \multicolumn{2}{|c|}{$\begin{array}{c}\text { Disfunción } \\
f(\%)\end{array}$} & \multicolumn{2}{|c|}{$\begin{array}{c}\text { Rechazo } \\
f(\%)\end{array}$} & Valor $p$ \\
\hline & & & & & \\
\hline Diabetes mellitus & 7 & $(29.2)$ & 47 & $(50.0)$ & \\
\hline Hipertensión arterial & 9 & $(37.5)$ & 20 & $(21.3)$ & \\
\hline Otros & 1 & $(4.2)$ & 7 & $(7.4)$ & \\
\hline Ninguna & 7 & $(29.2)$ & 20 & $(21.3)$ & \\
\hline Etiología de la enfermedad renal & & & & & 0.711 \\
\hline Diabetes mellitus & 0 & $(0.0)$ & 2 & $(2.1)$ & \\
\hline Enfermedad glomerular & 0 & $(0.0)$ & 2 & $(2.1)$ & \\
\hline Enf. renal poliquística/Tumores & 1 & $(4.2)$ & 2 & $(2.1)$ & \\
\hline Hipoplasia renal & 1 & $(4.2)$ & 8 & $(8.5)$ & \\
\hline Etiología no determinada & 22 & $(91.7)$ & 80 & $(85.1)$ & \\
\hline Terapia de reemplazo renal & & & & & 0.805 \\
\hline Diálisis & 3 & $(12.5)$ & 13 & $(13.8)$ & \\
\hline Hemodiálisis & 9 & $(37.5)$ & 41 & $(43.6)$ & \\
\hline Diálisis y Hemodiálisis & 12 & $(50.0)$ & 40 & $(42.6)$ & \\
\hline Toxicomanías & & & & & 0.653 \\
\hline Alcoholismo & 1 & $(4.2)$ & 4 & $(4.3)$ & \\
\hline Tabaquismo & 2 & $(8.3)$ & 18 & (19.1) & \\
\hline Ambas & 1 & $(4.2)$ & 4 & $(4.3)$ & \\
\hline Ninguno & 20 & $(83.3)$ & 68 & $(72.3)$ & \\
\hline Hacinamiento & & & & & 0.919 \\
\hline Si & 13 & $(54.2)$ & 52 & $(55.3)$ & \\
\hline No & 11 & $(45.8)$ & 42 & (44.7) & \\
\hline Peso (Kg)* & & $8 \pm 9.3$ & & $63.0 \pm 9.9$ & 0.585 \\
\hline Talla $(\mathrm{cm})^{*}$ & & $2 \pm 0.07$ & & $1.63 \pm 0.09$ & 0.322 \\
\hline $\operatorname{IMC}\left(\mathrm{Kg} / \mathrm{m}^{2}\right)^{*}$ & & $2 \pm 5.0$ & & $23.1 \pm 3.3$ & 0.387 \\
\hline
\end{tabular}

Comparación de frecuencias a través de la prueba chi cuadrada.

${ }^{*}$ Se presentan como media y desviación estándar.

\begin{tabular}{|c|c|c|c|c|c|}
\hline \multirow{2}{*}{ Tipo de donador } & \multicolumn{2}{|c|}{$\begin{array}{l}\text { Disfunción } \\
\text { f (\%) }\end{array}$} & \multicolumn{2}{|c|}{$\begin{array}{c}\text { Rechazo } \\
\mathrm{f}(\%)\end{array}$} & \multirow{2}{*}{$\begin{array}{c}\text { Valor } p \\
0.871\end{array}$} \\
\hline & & & & & \\
\hline Donador vivo & 8 & (33.3) & 33 & (35.1) & \\
\hline Donador fallecido & 16 & (66.7) & 61 & (64.9) & \\
\hline Parentesco con el donador & & & & & 0.339 \\
\hline Padre/Madre & 9 & (33.3) & 15 & $(16.0)$ & \\
\hline Hermano(a) & 0 & $(0.0)$ & 18 & (19.1) & \\
\hline Ninguno & 16 & (66.7) & 61 & (64.9) & \\
\hline $\begin{array}{l}\text { Tiempo transcurrido desde el trasplante } \\
\text { (años)* }\end{array}$ & & $2.4 \pm 1.7$ & & $2.4 \pm 1.9$ & 0.965 \\
\hline Tiempo de isquemia fría (horas)* & & $10.5 \pm 6.5$ & & $12.4 \pm 9.7$ & 0.375 \\
\hline
\end{tabular}

Comparación de frecuencias a través de la prueba chi cuadrada.

*Se presenta como media y desviación estándar. 
Adicionalmente, se sugiere continuar con el seguimiento de los pacientes que recibieron un trasplante y llevar a cabo las estrategias necesarias para garantizar mejores resultados posteriores al TR7. Günay et al.4, siguieron a receptores renales durante 10 años y observaron que el 29.5\% de los pacientes habían presentado pérdida de injerto. Además, se encontró que el rechazo recurrente, la disfunción crónica ${ }^{26}$ y la muerte del paciente con injerto funcional ${ }^{27}$ fueron las principales causas de pérdida de injerto a largo plazo.

Finalmente, es importante reconocer las limitaciones del presente estudio, como la falta de información respecto a las características del donante fallecido (información confidencial no reportada en el expediente clínico) que pudieran relacionarse con la presencia de complicaciones posteriores al TR. Como ejemplo se encuentra la FRI, definida como la necesidad de diálisis dentro de la primera semana después del trasplante y que se asocia con un mayor riesgo de pérdida de injerto y muerte con un injerto funcional. Melih et al.$^{28}$, identificaron factores de riesgo predictores de la FRI como el tiempo de isquemia fría, la edad del donante y el donante marginal. Por su parte Muth et al. ${ }^{29}$, implementaron una clínica multidisciplinaria dirigida por personal de enfermería para el manejo ambulatorio de los pacientes con FRI, con lo que lograron una disminución en la incidencia de rechazo agudo.

\section{CONCLUSIONES}

La disfunción y el rechazo de injerto renal continúan siendo problemas frecuentes posteriores al TR. Aunque no es el caso del presente estudio, se ha encontrado asociación entre algunas características sociodemográficas y la presencia de complicaciones, por lo que se sugiere establecer estrategias e intervenciones de enfermería para mejorar la supervivencia del paciente y del injerto renal.

\section{RESPONSABILIDADES ÉTICAS}

Protección de personas y animales. Los autores declaran que en el presente estudio no se realizaron experimentos en seres humanos ni animales.

Confidencialidad. En este estudio no aparecen datos que permitan identificar a los participantes.

Derecho a la privacidad y consentimiento informado. Los autores declaran que han firmado carta de confidencialidad.

Conflicto de intereses. Los autores declaran no tener conflicto de intereses.

Financiamiento. Ninguno.

Agradecimientos. A las autoridades por el apoyo recibido y personal del Dpto. de estadística.

\section{REFERENCIAS}

1. López-Falcony R, Ramírez-Orozco RE, Rodríguez-Jamaica JS, Fuentes-García P. Detección de microalbuminuria y su asociación con el índice de masa corporal en asistentes al escuadrón de protección renal. Rev Mex Traspl. 2016; 5(1): 15-9.

2. Gonzalez-Bedat MC, Rosa-Diez G, Ferreiro A. El registro latinoamericano de diálisis y trasplante renal: la importancia del desarrollo de los registros nacionales en latinoamérica. Nefrol Latinoam. 2017; 14 (1): 12-21. https://doi.org/10.1016/j.nefrol.2016.12.002

3. Secretaría de Salud / Centro Nacional de Trasplantes. Reporte anual 2019 de donación y trasplantes en México. México: SSA / CENATRA; 2019. https://bit.ly/3mU1Txg

4. Günay E, Çelebi T, Şen S, Aşcı G, Sarsik Kumbaraci B, Gökalp C, et al. Investigation of the factors affecting allograft kidney functions: Results of 10 years. Transplant Proc. 2019; 51(4): 1082-5.

https://doi.org/10.1016/j.transproceed.2019.02.014 
5. Rebello-Bicalho P, Requião-Moura LR, Ferraz-Arruda É, Chinen R, Mello L, Bertocchi APF, et al. Longterm outcomes among kidney transplant recipients and after graft failure: A single-center cohort study in Brazil. Biomed Res Int. 2019. https://doi.org/10.1155/2019/7105084

6. Baker RJ, Marks SD. Management of chronic renal allograft dysfunction and when to re-transplant. Pediatr Nephrol. 2019; 34(4): 599-603. https://doi.org/10.1007/s00467-018-4000-9

7. Campistol JM, Gutiérrez-Dalmau A, Crespo J, Saval N, Grinyó JM, Grupo de Estudio Observa. Actitud clínica frente a la disfunción renal en receptores de un trasplante renal en España. Nefrología. 2015; 35(3): 256-63. http://dx.doi.org/10.1016/j.nefro.2015.05.006

8. Seija M, Nin M, Astesiano R, Coitiño R, Santiago J, Ferrari S, et al. Rechazo agudo del trasplante renal: diagnóstico y alternativas terapéuticas. Nefrol Latinoam. 2017; 4(4): 119-30.

https://doi.org/10.1016/j.nefrol.2017.10.001

9. Etta PK, Rao MV. Renal allograft dysfunction: An update on immunological graft injury. Indian J Transplant. 2019; 13(2): 69 77. https://doi.org/10.4103/ijot.ijot_84_18

10. Moreso F, Hernández D. ¿Ha mejorado la supervivencia del injerto tras el trasplante renal en la era de la moderna inmunosupresión? Nefrología. 2013; 33(1): 14-26.

https://doi.org/10.3265/Nefrologia.pre2012.Oct.11739

11. Ruiz-Calzado MR. La adherencia terapéutica en el paciente trasplantado renal. Enferm Nefrol. 2013; 16(4): 258-70. http://dx.doi.org/10.4321/S2254-28842013000400007

12. Durán-Muñoz MI, Lope-Andrea T, del Pino-Jurado MR, Chicharro-Chicharro MC, Matilla-Villar E. Adherencia al tratamiento inmunosupresor en el paciente adulto con trasplante renal. Enferm Nefrol. 2013; 15(4): 300-5. http://dx.doi.org/10.4321/S2254-28842012000400010

13. Magott-Procelewska M, Boratyńska M, Madziarska K, Chudoba P, Klinger M. Impaired kidney allograft: How long can it function? A single-center study of the transplantation population. Transplant Proc. 2014; 46(8): 2692-5. https://doi.org/10.1016/j.transproceed.2014.08.022

14. Mahendran AO, Barlow AD. Kidney transplantation. Surgery. 2014; 32(7):364-70.

https://doi.org/10.1016/j.mpsur.2014.04.010

15. Haas M, Loupy A, Lefaucheur C, Roufosse C, Glotz D, Seron D, et al. The Banff 2017 kidney meeting report: Revised diagnostic criteria for chronic active $\mathrm{T}$ cell-mediated rejection, antibody-mediated rejection, and prospects for integrative endpoints for next-generation clinical trials. Am J Transplant. 2018; 18(2): 293-307. https://doi.org/10.1111/ajt.14625

16. Asociación Médica Mundial. Declaración de Helsinki de la AMM-Principios éticos para las investigaciones médicas en seres humanos. Riesgos , costos y beneficios. Francia: AMM; 2017.

17. Secretaría de Salud. Reglamento de la Ley General de Salud en materia de investigación para la salud. México: DOF; Última reforma 2014.

18. Gorayeb-Polacchini FS, Caldas HC, Gauch CR, Ferreira-Baptista MAS, Fernandes-Charpiot IMM, Abbud-Filho M. Factors that influence delayed graft function in kidney transplants: A single-center paired kidney analysis. Transplant Proc. 2019; 51(5): 1568-70.

https://doi.org/10.1016/j.transproceed.2019.01.040

19. Hansson J, Mjörnstedt L, Lindnér P. The risk of graft loss 5 years after kidney transplantation is increased if cold ischemia time exceeds 14 hours. Clin Transplant. 2018; 32(9): 1-27.

https://doi.org/10.1111/ctr.13377

20. Gong L, Wang K. Research progress on preventions of ischemia-reperfusion injury during kidney transplant. Sheng Wu Yi Xue Gong Cheng Xue Za Zhi. 2018; 35(5): 817-21.

https://doi.org/10.7507/1001-5515.201804009 
21. Stolyar AG, Tomilina NA. Impact of smoking on kidney transplantation outcomes. Ter Arkh. 2016; 88(12): 45-50. https://doi.org/10.17116/terarkh2016881245-50

22. Erturk T, Berber I, Cakir U. Effect of obesity on clinical outcomes of kidney transplant patients. Transplant Proc. 2019; 51(4): 1093-5. https://doi.org/10.1016/j.transproceed.2019.02.012

23. Liese J, Bottner N, Büttner S, Reinisch A, Woeste G, Wortmann M, et al. Influence of the recipient body mass index on the outcomes after kidney transplantation. Langenbecks Arch Surg. 2018; 403(1): 73-82. https://doi.org/10.1007/s00423-017-1584-7

24. Tzvetanov I, D’Amico G, Walczak D, Jeon H, Garcia-Roca R, Oberholzer J, et al. High rate of unemployment after kidney transplantation: Analysis of the united network for organ sharing database. Transplant Proc. 2014; 46(5): 1290-4. http://dx.doi.org/10.1016/j.transproceed.2014.02.006

25. Pistorio ML, Veroux M, Trigona C, Patanè M, Lo Bianco S, Cirincione G, et al. Psychological and Emotional Aspects in Living Donor Kidney Transplantation. Transplant Proc. 2019; 51(1): 124-7. https://doi.org/10.1016/j.transproceed.2018.04.085

26. Hamza WM, Ali HH, Gabal SM, Fadda SA. Chronic renal allograft dysfunction in Egyptian population: Histopathological and immunohistochemical Study. Saudi J Kidney Dis Transpl. 2016; 27(5): 921-8. https://doi.org/10.4103/1319-2442.190818

27. González-Posada Delgado JM, Pérez-Tamajón L, Marrero-Miranda D, Rodríguez-Hernández A, Delgado-Mallén P, Álvarez-González A, et al. Nefrología al día. Complicaciones médicas precoces tras el trasplante renal. Barcelona: Sociedad Española de Nefrología (S.E.N.); 2016.

https://bit.ly/32b60Sw

28. Melih KV, Boynuegri B, Mustafa C, Nilgun A. Incidence, risk factors, and outcomes of delayed graft function in deceased donor kidney transplantation. Transplant Proc. 2019; 51(4): 1096-100. https://doi.org/10.1016/j.transproceed.2019.02.013

29. Muth BL, Astor BC, Turk J, Mohamed M, Parajuli S, Kaufman DB, et al. Outpatient management of delayed graft function is associated with reduced length of stay without an increase in adverse events. Am J Transplant. 2016; 16(5): 1604-11. https://doi.org/10.1111/ajt.13689 